\title{
An industry update: what's new in the field of therapeutic delivery this month?
}

First draft submitted: 6 October 2016; Accepted for publication: 11 October 2016; Published online: 8 November 2016

This Industry Update covers the period from 1 to 30 September 2016, and is based on information sourced from company press releases, scientific literature, patents and various news websites. The month saw a number of major therapeutic conferences taking place including European Respiratory Society (ERS) International Congress (covering respiratory disease) and The European Committee for Treatment and Research in Multiple Sclerosis (ECTRIMS) Congress (covering multiple sclerosis) at which updates on clinical programs were given by AstraZeneca (LSE:AZN; London, UK), GlaxoSmitKline (LSE:GSK, London, UK) and Novartis (SIX:NOVN; Basel, CH). September was also Alzheimer's Awareness Month and brought positive results from Biogen (NASDAQ: BIIB, MA, USA) and AstraZeneca/Eli Lilly (NYSE:LLY; IN, USA), that they had received the US FDA fast track designation for their investigational drugs, which are both in Phase III development. Meanwhile Lundbeck (CPH:LUN; Copenhagen, Denmark) reported negative results for one of its Alzheimer's drugs. Takeda (TYO:4502; Osaka, Japan) announced funding from the US government to support its development of a vaccine for the Zika virus and Verily (formerly Google Life Sciences, part of Alphabet Inc, NASDAQ: GOOGL; CA, USA) announced another pharma deal, this time with Sanofi (EPA: SAN; Paris, France) to develop technology to support patients with diabetes. Aspen Holdings announced another deal to acquire assets in the anesthesia market, this time from AstraZeneca. Bristol-Myers Squibb (BMS, NYSE: BMY; NY, USA) and Nektar (NASDAQ: NKTR; CA, USA) announced a collaboration to look at the benefits of combining one of Nekar's investigational drugs with Bristol-Myers Squibb's already approved drug, Opdivo, for a range of oncology indications. Janssen (part of Johnson and Johnson, NYSE:JNJ; Beerse, Belgium) announced that it had submitted applications for market approval for a new rheumatoid arthritis drug both in the USA and Europe, and Sarepta (NASDAQ: SRPT; MA, USA) finally got conditional approval for its treatment for Duchenne muscular dystrophy. French start-up, Poietis (Privately held; Bordeaux, France) announced a deal to develop its 3D bioprinting technology for hair restoration with L'Oréal (EPA:OR, Paris, France). But perhaps more importantly, the technology may have much wider applications in drug testing and regenerative medicine.

\section{Business development}

\section{Takeda Pharmaceutical Company Ltd}

Although concerns about risks from the Zika virus at the Olympic Games recently held in Rio de Janeiro, Brazil appear to have been overstated, the search for a vaccine to treat a disease that is now prevalent in $>60$ countries, putting around two billion people at risk, continued with announcement that Takeda (TYO:4502; Osaka, Japan) has won up to US\$312 million in funding from the US government to develop a vaccine for use in the USA. An initial contract of US $\$ 19.8$ million covers work through to Phase I trials planned for 2017, with the potential for further funding to take the development to a Biologics License Application in the USA. Other potential Zika vaccines currently in early human testing lain Simpson

IXICO PLC, Griffin Court 15 Long Lane, London, EC1A 9PN, UK, iain.simpson@ixico.com 
are mainly DNA-based and contain no actual virus, whereas the Takeda vaccine will aim to promote an immune response using inactivated whole Zika virus [1].

\section{Verily Life Sciences \& Sanofi}

Verily Life Sciences LLC (formerly Google Life Sciences, part of Alphabet Inc, NASDAQ: GOOGL; CA, USA) continued its foray into life sciences with an announcement that it is setting up a joint venture with Sanofi SA (EPA: SAN; Paris, France) to help people with diabetes lead more normal lives. The joint venture, called Onduo, will develop technology that combines devices with software, medicine and professional healthcare support to provide simple and intelligent disease management. Onduo combines Sanofi's clinical expertise in developing therapeutics to treat diabetes, with Verily's expertise in software, miniaturized electronics and data analytics.

Onduo will initially focus on Type 2 diabetes, developing solutions that allow patients to make better informed choices about their day to day healthcare management. In longer term, the company intends to expand into Type 1 diabetes [2].

This is an example of an industry trend to develop more comprehensive disease management solutions that combine therapeutics with devices and software to develop 'digital services'.

\section{Aspen \& AstraZeneca}

Aspen Pharmacare Holdings Ltd (JSE: APN; Johannesburg, South Africa) announced that it has concluded an agreement to acquire global commercialization rights (outside the USA) for a portfolio of seven established anesthetic drugs from AstraZeneca PLC (LSE:AZN; London, UK), for an initial payment of US $\$ 520$ million plus double-digit royalties. The rationale for Aspen is it allows it to expand the portfolio of drugs that its hospital-focused sales team can offer alongside its existing anticoagulants. For AstraZeneca, this is an opportunity to realize revenues from noncore products while maintaining a focus in developing new therapies in its core areas such as oncology and respiratory disease. It has seen a significant decline in its share of anesthetics market over the past few years, and considers the genericized nature of this market and its emphasis on hospital sales as not being a good fit with AZN's focus on novel therapeutics marketed directly to doctors.

In a similar deal, GlaxoSmitKline (GSK) announced that it is also divesting its anesthesia portfolio to Aspen for $\mathrm{GB} £ 180$ million plus up to a further $\mathrm{GB} £ 100$ million in future milestone payments. It also announced its intention to sell its remaining equity stake to Aspen [3].
Bristol-Myers Squibb \& Nektar Therapeutics Bristol-Myers Squibb Company (BMS, NYSE: BMY; NY, USA) and Nektar Therapeutics (NASDAQ: NKTR; CA, USA) announced a collaboration to develop an oncology combination treatment utilizing BMS's already approved drug Opdivo with a Nektar drug candidate NKTR-214. The announcement follows disappointing Phase III results published in August 2016 for Opdivo in the treatment of lung cancer. At that time, BMS commented that the future of Opdivo was likely to be built upon the development of combination therapies, so this announcement is a step in that direction. The collaboration plans to conduct studies with a range of tumor types including lung cancer. Phase I/II open-label data should start to be available in 2018, which will then allow the strategy to get to product launch to be more clearly defined.

Opdivo is a monoclonal antibody (mAb), which works as a checkpoint inhibitor allowing $\mathrm{T}$ cells to identify and attack cancer cells, which would otherwise be 'masked'. In December 2014, it was approved by the US FDA for the treatment of melanoma and for further indications since then. Although it has similar mode of action to Keytruda, marketed by Merck, which is also a PD-1 inhibitor, the two companies have adopted different market strategies, with Opdivo being marketed more broadly whereas Keytruda is used with a diagnostic test to assess if the drug is likely to be effective for a particular tumor. Avoiding the need for a test has been one factor in Opdivo having greater sales than Keytruda but as more clinical data are gathered, the potential of greater efficiency for the latter, given the biomarker test may turn the tables, hence the comments by BMS about looking at combination products and the recent announcement of the deal with Nektar [4].

NKTR-214 is an experimental therapy that aims to stimulate cancer-killing immune cells in the body by targeting specific receptors on their surface, and hence triggering a stronger immune response. In addition to the evaluation of its performance in conjunction with Opdivo, Nektar also intends to look at its effectiveness as an oncology monotherapy [5].

\section{Development \& clinical trials \\ AstraZeneca}

AstraZeneca PLC published results of a Phase III pivotal trial with the $\mathrm{mAb}$ benralizumab at the recent ERS International Congress, held in London, UK, which it claimed showed significantly reduced exacerbations and improved lung function in patients with severe asthma. A total of 2511 patients already receiving standard of care medicine (including high-dosage inhaled corticosteroids [ICSs] and long-acting $\beta 2$ agonist) were 
enrolled in the randomized study and received by subcutaneous injection, either benralizumab in 4- or 8-week dosing regimens (after an initial induction period) or placebo over periods of up to 56 weeks. The data showed a reduction in asthma exacerbations and an improvement in lung function (as measured by FEV1, a standard measure for asthma). An improvement in other common asthma symptoms such as shortness of breath, cough and chest tightness was also observed. The company also noted that the less frequent 8 -week dosing might be as effective as the 4-week regimen. It hopes to file for approval of the drug in the USA and the EU later this year. In the respiratory disease market, there is a move from inhaled small molecules toward injected biologic therapies, particularly mAbs for underlying treatment of asthma with Novartis' Xolair, GSK's Nucala and Teva's (NYSE:TEVA; Petah Tikva, Israel) Cinqair already approved in a number of territories. Nucala, Cinqair and benralizumab all target eosinophilic inflammation and are likely to come head to head in seeking a share of an increasingly crowded market for asthma therapeutics [6].

\section{GSK}

Also at the ERS International Congress, GSK (LSE:GSK, London, UK) presented further data from its Phase III trial with triple combination therapy fluticasone furoate/umeclidinium/vilanterol (FF/UMEC/VI) in patients with chronic obstructive pulmonary disease (COPD). The study puts the combination drug headto-head with AZN's marketed Symbicort ${ }^{\circledR}$ Turbohaler $^{\circledR}$ (budesonide/formoterol) in patients with advanced COPD. Results showed statistically significant improvements both in lung function and quality of life measures compared with Symbicort. Importantly, the study also showed a reduction in the annual rate of exacerbations compared with Symbicort (35 and 44\% for the 24- and 52-week data, respectively). Exacerbations are seen to be major source of morbidity for COPD sufferers.

FF/UMEC/VI combines an ICS (FF), an anticholinergic (UMEC), also known as a long-acting muscarinic and a long-acting $\beta_{2}$ adrenergic agonist (VI), and is delivered once-daily in GSK's Ellipta ${ }^{\circledR}$ dry powder inhaler, which is already in the market with other respiratory disease therapies. Symbicort Turbohaler (Turbuhaler in some countries) was originally approved in a number of markets for asthma and in 2003 was approved in Europe as a maintenance therapy of COPD in patients with severe COPD and a history of exacerbations. It was approved in the USA for COPD in 2009 but in a pressurized metered dose inhaler format. Unlike the FF/UMEC/VI combination, it is administered twice rather than once per day.

Back in June, GSK announced that following discussions with the FDA, it now expects to file for approval in later 2016 rather than in 2018, which was the previous expectation [7].

\section{Novartis}

In another piece of news from the ERS congress, Novartis International AG (SIX:NOVN; Basel, CH) presented data showing that its Ultibro ${ }^{\circledR}$ Breezhaler $^{\circledR}$ reduced the rate of all COPD exacerbations across different patient subgroups compared with GSK's Seretide product. There are increasing concerns over the risks of using ICSs, so it is particularly notable that patients using Ultibro needed to resort less often to rescue therapies, often ICSs, which are taken to address exacerbations and are also an indication of poor control of the disease. Novartis also claims safety benefits, as use of Ultibro Breezhaler in the study was associated with fewer systemic effects, such as impairment of adrenal function, which can affect and previous data has already shown lower rates of pneumonia compared with the use ICS/long-acting $\beta$ agnostic (LABA) combinations.

Ultibro Breezhaler is a once-daily combination of indacaterol and glycopyrronium bromide, respectively, a LABA and a long-acting muscarinic antagonist delivered once per day using the Breezhaler capsule inhaler. The product is approved in Europe and launched in around 90 countries around the world. Seretide, which is a combination of fluticasone and salmeterol, respectively an ICS and an LABA, became the world's largest respiratory drug with annual sales peaking at US $\$ 8.7$ billion in 2011 for indications covering both asthma and COPD. Although sales have declined due to generic entrants and newly approved therapies, they are still around US $\$ 5$ billion annually [8].

\section{Novartis}

At the The European Committee for Treatment and Research in Multiple Sclerosis (ECTRIMS) Congress recently held in London, UK, Novartis reported promising Phase II results for BAF312 (siponimod), once a day (oral), being developed for the treatment of secondary progressive multiple sclerosis (SPMS). Results from the study of 1651 people from 31 countries with SPMS showed reduced risk of 3-month confirmed disability progression by $21 \%$ compared with the placebo group. Around $85 \%$ of multiple sclerosis (MS) sufferers are initially diagnosed with relapsing remitting MS, which is the stage at which most current drugs are effective, but most patients eventually progress into SPMS, which is more debilitating and difficult to manage with existing therapies. Novantrone (mitoxantrone) is currently the only medication specifically approved by the FDA for SPMS despite it being a strong immunosuppressant originally developed to treat cancer and having signifi- 
cant side effects that limit its use. So Novartis could be well positioned to achieve blockbuster status in a key market with limited competition, which is timely given the looming generic threat to one of its existing drugs Gilenya, which has annual sales in excess of US $\$ 2$ billion. Its Swiss rival Roche (SIX: ROG; Basel, Switzerland) is developing another MS drug, Ocrevus, but this is targeting relapsing remitting MS as well as the $15 \%$ of patients who are initially diagnosed with primary progressive MS rather than SPMS [9].

\section{Omeros Corporation}

Omeros (NASDAQ: OMER; WA, USA) reported clinical results from its OMS721 development program and showed potential in treating acute ischemic brain injury or stroke. OMS 721 is a human mAb targeting MASP-2. MASP-2 plays a role in the inflammatory response and becomes activated as a result of tissue damage or microbial infection.

The Omeros' sponsored study shows that MASP-2-deficient mice have significantly reduced infarct volumes (one of the common measures for assessing the extent of ischemic brain injury) compared with normal mice following ischemia. Using a derivative of OMS721 modified for improved activity in mice, and administered to naive mice in three doses, two prior to and the third following ischemia, resulted in a similar reduction to infarct volume compared with the MASP-2 deficient mice. The data, therefore, appear to identify MASP-2 as a primary driver in ischemic brain tissue injury.

Omeros has a number of clinical programs utilizing OMS272 covering atypical hemolytic uremic syndrome, which is an extremely rare, life-threatening, progressive disease and for IgA nephropathy and other renal diseases, which are currently in Phase III and Phase II studies [10].

\section{Regulatory news \& approvals Biogen, Inc.}

September is World Alzheimer's Month, an international campaign to raise awareness of the disease and call for action to develop effective treatments. So it is particularly timely that Biogen (NASDAQ: BIIB, MA, USA) should announce that its investigational drug for early Alzheimer's disease (AD), aducanumab, has been granted Fast Track designation by the FDA. This designation is awarded to drugs that are seen to address serious conditions, such as $\mathrm{AD}$, where there is high unmet need. Biogen recently published preclinical and Phase Ia data in Nature, which demonstrated reduced amyloid- $\beta$ $(\mathrm{A} \beta)$ in the brain, a key biomarker for AD. Most significantly, those treated at the highest doses showed almost complete clearance of the $A \beta$ plaques. These Phase Ia studies were completed a few years back but the results are now being published and the drug is currently in Phase III studies focused on assessing its ability to slow cognitive impairment and the progression of disability in people with early stage $\mathrm{AD}$. Aducanumab is a human recombinant $\mathrm{mAb}$ derived from $\mathrm{B}$ cells from healthy elderly volunteers using a technology called Reverse Translational Medicine developed by Biogen's partner Neurimmune Holding AG (Schlieren, CH). Its mode of action is to bind selectively to the $A \beta$ plaques, which are then eliminated by the body's own phagocytes [11].

This follows recent announcement by AstraZeneca and Eli Lilly (NYSE:LLY; IN, USA), that they have also gained FDA Fast Track designation for another drug being developed to treat AD, AZD3293, an orally available BACE inhibitor, which is also in Phase III development. BACE inhibitors work in a different way to antibodies such as aducanumab; their mode of action is to stop cleavage of amyloid precursor proteins by enzymatic action, and hence stop $A \beta$ plaques forming in the first place [12].

There was less promising news for $\mathrm{H}$ Lundbeck AS (Lundbeck) (CPH: LUN; Copenhagen, Denmark), which announced poor results for a Phase III study for its investigational Alzheimer's Lu AE58054 when administered alongside Donepezil (Aricept). No significant benefit over placebo treatment was seen over placebo in a study involving over 900 patients with mild-to-moderate AD [13].

\section{Janssen Biotech, Inc. (Johnson \& Johnson)}

In September, Janssen Biotech, Inc. (a division of Janssen Pharmaceutical Companies of Johnson \& Johnson, NYSE:JNJ; Beerse, Belgium) announced that it has submitted applications to both the FDA and EMA for approval of sirukumab for the treatment of adult patients with moderately to severely active rheumatoid arthritis (RA). The company with its partner, GSK initiated a number of Phase III studies including those with patients inadequately treated with diseasemodifying antirheumatic drugs such as methotrexate as well as those not responding to treatment with anti-TNF agents and other biologic agents. Published results show positive outcomes for these patient groups. However, if approved, the drug will face stiff competition from existing drugs such as Humira (one of a number of marketed anti-TNF agents and the world's largest selling drug by value) as well as another $\mathrm{mAb}$, being developed by Sanofi and Regeneron, sarilumab, which is already filed for approval in the USA and the EU. Notably, preliminary head-to-head data for sarilumab against Humira (adalimumab) have already been presented, which shows superiority. Janssen and GSK are also conducting similar head-to-head stud- 
ies for sirukumab against Humira but results have yet to be presented. NIHR is suggesting that if approved, sirukumab is likely to be used as a second- or thirdline treatment for patients with moderate to severe $\mathrm{RA}$, who are not responding to existing drugs such as methotrexate or Humira, which will limit sirukumab's market.

Sirukumab is a human $\mathrm{mAb}$ that selectively binds to the IL-6 cytokine, a naturally occurring protein that is believed to play a role in autoimmune conditions such as RA.

After initial development work, Janssen had entered into a licensing and co-development agreement of sirukumab with GSK prior to initiation of Phase III studies that commenced in 2012 for RA.

The drug has also been evaluated for treating lupus nephritis, and showed poor results. It is also undergoing on-going studies for asthma and giant cell arteritis - a form of vasculitis [14].

\section{Sarepta Therapeutics}

Sarepta Therapeutics (NASDAQ: SRPT; MA, USA) received FDA approval for its drug EXONDYS 51 ${ }^{\mathrm{TM}}$ (eteplirsen) injection, for treatment of Duchenne muscular dystrophy (DMD). The drug was approved under the accelerated approval pathway, which provides for the approval of drugs that treat serious or life-threatening diseases, which are currently inadequately treated; to date, there is no approved drug for the treatment of DMD.

Despite the eventual fast track approval, Sarepta has had a number of setbacks in the review process. Back in April, an FDA review panel of external experts voted seven to six that there was insufficient evidence to establish that the drug provided clinical benefit. In the end, the FDA concluded that the observed dystrophin production is reasonably likely to predict clinical benefit in some patients with DMD but has called for further clinical data to show clinical benefit in terms of improved motor function with an agreed final reporting date of May 2021. Depending on the outcome of these studies the FDA could withdraw approval.

DMD is the most common type of muscular dystrophy affecting about 1 in 5000 males at birth and with an average life expectancy for sufferers of 26 years. Onset of symptoms is typically around the age of 4 years with most sufferers being unable to walk by the age of 12 years.

DMD is caused by a mutation in the gene for the protein dystrophin, which is important in maintenance of the membranes of muscle fiber cells. This most commonly occurs genetically but can also be caused by new mutations. EXONDYS 51 is only effective for sufferers who have a confirmed mutation of the dystrophin gene amenable to exon 51 skipping, which is around 13\% of DMD sufferers. During dystrophin synthesis EXONDYS 51 binds to exon 51 and thus allows encoding to skip this exon and produce the protein [15].

BioMarin (NASDAQ: BMRN; CA, USA) was also in the hunt for approval for an Exon 51 skipping drug, Kyndrisa ${ }^{\mathrm{TM}}$ (drisapersen), following its acquisition of Prosensa in 2014, and had filed for approval with the FDA, but in June 2016 following receipt of a competed response letter from the FDA and discussions with the EMA decided to abandon all development of the drug and instead focus on other approaches to treating the disease.

It is worth noting that this approval qualifies Sarepta for a rare pediatric disease priority review voucher from the FDA, which allows for a future submission to be fast tracked. These vouchers can be sold to another party and are proving to be valuable with United Therapeutics Corporation selling one to AbbVie for US $\$ 350$ million and Sanofi purchase of a priority review voucher from BioMarin for $\$ 67$ million, and used its fast track approval for its drug Praluent in its race with Amgen (NASDAQ: AMGN; CA, USA) to be the first to market with PCSK9 inhibitor.

\section{Market news}

\section{Novartis}

Novartis announced that it had gained a CE mark for a new point of care system NijiTM that can provide earlier diagnosis and faster treatment decisions for severe allergic asthma, which is a major area of therapeutic focus for the company, as well as for other diseases.

Tests can be completed within 12 min using blood samples collected using a finger stick test similar to that used by diabetics, allowing for quick in-office diagnosis of IgE-mediated allergic disorders, such as asthma. Results from conventional IgE tests using centralized labs can take weeks to be delivered and are thus too slow for rapid diagnosis and action. The new test allows for result to be gathered and assessed in a single session and along with other clinical findings allow for better treatment decisions to be made in a single consultation.

Novartis plans to launch the system in Europe later this year and considers the system to be a platform for fast and easy blood tests that could potentially be applied across a variety of disease areas [16].

The technology platform was originally developed by the company Vivacta, which was bought by Novartis for US\$90 million in 2012. 


\section{Vectura}

Vectura PLC (LSE:VEC.L; Chippenham, UK) provided a trading update this month and highlighted significant growth in sales for Flutiform (fluticasone propionate/formoterol fumarate dihydrate), an ICS-LABA combination therapy approved for the treatment of asthma and marketed by Mudipharma in Europe and Kyorin in Japan. The drug was developed by Skyepharma, which was acquired by Vectura earlier in the year. Sales for the 6 -month period to 30 June 2016 were up $42 \%$ on the same period in 2015 and the drugs are now launched in 31 countries, approved in a further nine and under review for approval in 15 others. The company also reported good sales growth from GSK's Ellipta respiratory products revenues as well as for Novartis' Seebri and Ultibro Breezhaler products, all products for which it receives licensing revenues. It also announced the results of a product pipeline review that it has been conducting following the acquisition and integration of Skyepharma [17].

\section{Mylan}

Concerns about the pricing of Mylan's (NASDAQ: MYL; PA, USA) (epinephrine injection, USP) autoinjector continued to rumble with an announcement that New York's Attorney General has launched an investigation into supply of the drug. It is alleged that Mylan's sales contract with a local school system may have included 'potentially anticompetitive terms' and comes revelations that the price of EpiPen has increased by fivefold since 2007 when Mylan acquired Dey Pharmaceuticals from Merck.

Furthermore, it has also been stated in the press that while the price of an EpiPen two-pack has risen to more than US\$600 in the National Health Service in the UK only pays around US $\$ 69$ for the same product [18].

Mylan has responded to the pricing furore by offering a lower priced generic version of EpiPen at around half the full price and has claims of significant investment in product development over the past few years. With the withdrawal of AuviQ by Sanofi and the delays for Teva getting approval for its generic version of EpiPen following FDA review, Mylan has a near monopoly on the epinephrine emergency market in the USA.

In election year in the USA, drug pricing is likely to come under even closer scrutiny over the coming months and once a new president is in office in 2017 [19].

\section{Patents \& novel technology}

Mylan

Mylan N.V. continues to challenge Teva's (Teva Pharmaceutical Industries Ltd, TLV: TEVA; Petah Tikva, Israel) patents covering its $40 \mathrm{mg} / \mathrm{ml}$ formulation of blockbuster multiple sclerosis (MS) drug Copaxone ${ }^{\circledR}$ and announced that a third patent has had all its claims invalidated by the US patent office. Teva got FDA approval for Copaxone $40 \mathrm{mg} / \mathrm{ml}$ back in 2014, as part of its defence of the drug from generic entrants. This formulation allows less frequent administration of the drug than the previous $20 \mathrm{mg} / \mathrm{ml}$ formulation (three-times per week rather than daily), and Teva in the USA succeeded in switching around $80 \%$ of Copaxone scripts, thus protecting a global franchise of around US\$4 billion, despite approval of Sandoz (Novartis), Momenta's (Momenta Pharmaceuticals, Inc., NASDAQ:MNTA; MA, USA) generic $20 \mathrm{mg} / \mathrm{ml}$ version in the USA in 2015 and Europe in 2016. Teva hoped that patents on the new formulation would extend protection out until 2030 but its position in the USA now looks threatened [20].

However, the situation for Teva in Europe looks better as in late 2015, the European Patent Office upheld a patent following a challenge by Mylan, Synthon Pharmaceuticals Inc. (Nijmegen, NL) and Actavis PLC (Now Allergan PLC, NYSE:AGN; NJ, USA) [21].

\section{Poietis \& L'Oreal}

French start-up Poietis (privately held; Bordeaux, France) announced a research partnership with cosmetics company L'Oreal (EPA:OR, Paris, France) to develop technology for the bioprinting of hair follicles, which could eventually be used in hair restoration.

Poietis, founded in September 2014, acquired the exclusive worldwide license for patents associated with the laser-assisted bioprinting technology from the University of Bordeaux. It claims that the method can deposit cellular material in 3D patterns with ten-micron resolution and high cell viability (in excess of 95\%). The technique is analogous to $3 \mathrm{D}$ printing, which was originally developed by a number of companies and research intuitions for rapid prototyping of components using a variety of methods but is now seeing use in commercial manufacture in many industries including medical devices and aerospace. Poietis's approach involves successively layering microdrops of bioinks using a scanning laser beam. The living biological tissue created is then 'matured' for around 3 weeks before it can be used.

The company is looking to produce physiological models of skin for drug and cosmetics research in order to assess the toxicity and efficacy of new products or drug candidates. They also envisage developing autologous grafts by harvesting cells from patients and then creating biological structures such as cartilage, cornea or skin, which can then be used with the same patient in regenerative medicine applications, reducing the risks of rejection or the need for immunosuppressant that are often required for transplants and artificial implants [22]. 
L'Oreal is already active in the use of $3 \mathrm{D}$ printing using printing in its product research.

\section{Financial \& competing interests disclosure}

The author has no relevant affiliations or financial involvement with any organization or entity with a financial interest in or

\section{References}

1 Takeda to develop Zika vaccine with up to $\$ 312$ million in funding from US government. www.takeda.com/news/2016/20160902_7524.html

2 Sanofi and Verily Life Sciences Announce Launch of Onduo, a Joint Venture to Develop Comprehensive Diabetes Management Platform mediaroom.sanofi.com/sanofi-and-verily-life-sciences

3 GSK divests non-core assets to Aspen. www.gsk.com/en-gb/media/press-releases/2016/gsk-divests

4 Bristol-Myers Squibb and Nektar therapeutics announce oncology clinical collaboration to evaluate the combination of Opdivo (nivolumab) and NKTR-214. www.prnewswire.com/news-releases/nektar-and-md

5 Nektar and MD Anderson Cancer center announce Phase I/II clinical research collaboration for NKTR-214, a CD122-biased immuno-stimulatory cytokine. www.prnewswire.com/news-releases/nektar-and-md

6 Benralizumab Phase III trials show positive results in severe asthma.

www.astrazeneca.com/media-centre/press-releases/2016

7 GSK presents positive results from phase III FULFIL study of closed triple combination therapy FF/UMEC/VI versus Symbicort ${ }^{\circledR}$ Turbohaler $^{\circledR}$ in COPD at ERS International Congress.

www.gsk.com/en-gb/media/press-releases/2016/gskpresents

8 Novartis' Ultibro ${ }^{\circledR}$ Breezhaler ${ }^{\circledR}$ consistently more effective than Seretide ${ }^{\circledR *}$ in reducing COPD flare-ups across different patient groups.

www.novartis.com/news/media-releases/novartis-ultibror

9 Novartis announces positive Phase III results showing efficacy of BAF312 in patients with secondary progressive MS.

www.novartis.com/news/media-releases/novartisannounces

10 Omeros' MASP-2 antibody preserves brain tissue and function in stroke models.

http://investor.omeros.com/phoenix financial conflict with the subject matter or materials discussed in the manuscript. This includes employment, consultancies, honoraria, stock ownership or options, expert testimony, grants or patents received or pending, or royalties.

No writing assistance was utilized in the production of this manuscript.

11 Biogen's investigational Alzheimer's disease treatment Aducanumab granted FDA fast track designation. http://media.biogen.com/press-release/neurodegenerative

12 AstraZeneca and Lilly receive FDA fast track designation for AZD3293, an investigational treatment for early Alzheimer's disease.

www.astrazeneca.com/media-centre/press-releases/2016/

13 Headline conclusions from the first out of three Phase III studies on idalopirdine in Alzheimer's disease. http://investor.lundbeck.com/releasedetail

14 Janssen submits application seeking approval of Sirukumab in European Union for rheumatoid arthritis. www.janssen.com/janssen-submits-application-seeking

15 Sarepta therapeutics announces FDA accelerated approval of EXONDYS $51^{\mathrm{TM}}$ (eteplirsen) injection, an exon skipping therapy to treat duchenne muscular dystrophy (DMD) patients amenable to skipping exon 51 . http://investorrelations.sarepta.com/phoenix

16 New Novartis point of care Niji(TM) system may provide earlier diagnosis of severe allergic asthma and faster treatment decisions. www.novartis.com/news/media-releases/new-novartis-point

17 AGM trading update. www.vectura.com/news/raisesexpectations-2016-revenues-and-announces-significantmerger-progress/

18 Why the $\$ 600$ EpiPen costs $\$ 69$ in Britain. www.bloomberg.com/news/articles/2016-09-29/epipen

19 New York investigates EpiPen maker Mylan after price hike of medication. www.theguardian.com/business/2016/sep/06/epipen-price

20 Mylan invalidates third Teva Copaxone ${ }^{\circledR} 40 \mathrm{mg} / \mathrm{ml}$ patent via US patent and trademark office's inter partes review proceeding. http://newsroom.mylan.com/2016-09-01-Mylan-Invalidates

21 Teva Copaxone patent upheld as valid by EPO. www.law360.com/articles/735404/corrected-teva-copaxone

22 L'Oréal and Poietis sign an exclusive research partnership to develop bioprinting of hair. www.poietis.com/eng/post-release. 